\section{Percepção de saúde e fatores associados em industriários de Santa Catarina, Brasil}

\author{
Self-perceived health and associated factors in \\ industrial workers from Santa Catarina State, Brazil
}

\author{
${ }_{1}$ Departamento de Ciências \\ da Saúde, Universidade \\ Estadual de Santa Cruz \\ Ilhéus, Brasil. \\ 2 Programa de Pós-Graduação \\ em Saúde Pública, \\ Universidade Federal \\ de Santa Catarina, \\ Florianópolis, Brasil. \\ 3 Grupo de Pesquisa em \\ Estilos de Vida e Saúde, \\ Universidade de Pernambuco, \\ Recife, Brasil. \\ 4 Programa de Pós-Graduação \\ em Educação Física, \\ Universidade Federal \\ de Santa Catarina, \\ Florianópolis, Brasil. \\ Correspondência \\ S. A. Fonseca \\ Departamento de Ciências \\ da Saúde, Universidade \\ Estadual de Santa Cruz. \\ Rodovia Ilhéus-Itabuna Km \\ 16. Campus Soane Nazaré \\ de Andrade, Ilhéus, $B A$ \\ 45662-000, Brasil. \\ silviofonsecatoledo@yahoo. \\ com.br
}

\begin{abstract}
The purpose of this study was to determine the prevalence of negative self-perceived health and associated factors among industrial workers in Santa Catarina State, in southern Brazil. A cross-sectional investigation was conducted with a representative sample of 2,574 subjects (62.5\% men). Negative self-perceived health (fair or poor) was the outcome investigated in association with demographic, socioeconomic, and other health indicators. Multivariate analysis was performed through logistic regression based on a hierarchical model. Negative self-perceived health was exceptional (11.8\%), but positively associated with age, leisure physical activity, perceived quality of sleeping, perceived stress, and sex. Meanwhile, the outcome was negatively associated with family income and schooling. Workers with higher physical demands and $B M I<18.5$ and $>30$ showed increased odds of negative self-perceived health. No significant differences were found for marital status, binge drinking, or smoking. Few associations were observed for females.
\end{abstract}

Occupational Health; Risk Factors; Perception
Silvio Aparecido Fonseca 1

Vera Lúcia Guimarães Blank 2

Mauro Virgílio Gomes de Barros 3

Markus Vinicius Nahas 4

\section{Introdução}

A associação da percepção de saúde com indicadores de mortalidade 1,2, comportamentos relacionados à saúde 3 , condições de vida 4,5,6 e de trabalho 7, tem sido reportada para diferentes populações. Estudos demonstram maior prevalência da percepção negativa de saúde em sujeitos com níveis elevados de estresse ${ }^{8}$, quantidade reduzida de sono, obesos ${ }^{9}$, fumantes ${ }^{5}$ e com baixo engajamento em atividades físicas de lazer 10 . Adicionalmente, mulheres 11 , pessoas de baixa escolarização 4 , de maior idade 6 , também referem pior nível de percepção de saúde.

Mediante essas evidências, em importantes estudos internacionais de base populacional $4,2,12$, e no recente inquérito domiciliar encomendado pelo Ministério da Saúde do Brasil 6, tem-se mensurado a autopercepção do nível de saúde por meio de uma pergunta simples, com opção de resposta em cinco escalas. Alternativamente, alguns estudos 8,11 utilizaram a pergunta com quatro opções de resposta (excelente, boa, regular e ruim), ainda que em estudo de Barros 13 sua reprodutibilidade tenha sido avaliada apenas como moderada $(k=0,59)$.

Adicionada à vantagem de aplicabilidade, esta estratégia para levantamento de informação acerca da saúde pode ser útil para complementar outros dados derivados da fase diagnóstica em programas para promoção da saúde de trabalhadores, permitindo uma análise mais crítica para 
o planejamento. Contudo, em âmbito nacional e internacional, poucas pesquisas 7,8,12 têm focado suas investigações na distribuição e nos determinantes da percepção de saúde deste grupo populacional. Mediante tal lacuna, o objetivo deste estudo é identificar a prevalência e os fatores associados à percepção negativa de saúde em trabalhadores da indústria no Estado de Santa Catarina, Brasil.

\section{Metodologia}

Utilizou-se a amostra representativa da população de 383.963 trabalhadores da indústria de Santa Catarina, participantes do estudo epidemiológico transversal Perfil do Estilo de Vida e Hábitos de Lazer de Trabalhadores da Indústria Catarinense/Brasil (PEVIC II), realizado no período de julho a setembro de 2004.

O processo de amostragem foi executado em estágios múltiplos, considerando as onze Unidades Regionais classificadas pelo SESI/DR-SC (Serviço Social da Indústria, Departamento Regional de Santa Catarina). No primeiro estágio, empregou-se a amostragem por julgamento 14 para a seleção de empresas de pequeno, médio e grande porte (10\% da fração de amostragem). O procedimento foi adotado por razões práticas e operacionais, bem como pela consideração dos autores da pesquisa e dos gestores do SESI/DR-SC, que as empresas selecionadas de forma aleatória estratificada no PEVIC I (1999) eram aquelas que ainda melhor representavam a população de industriários de Santa Catarina. Somente oito empresas se recusaram a participar, sendo substituídas aleatoriamente por outras do mesmo porte. Indústrias com menos de vinte empregados foram excluídas da pesquisa em função da possível variação e volatilidade na composição do número de funcionários.

Por fim, no segundo estágio, os sujeitos de cada empresa foram selecionados pela amostragem aleatória sistemática. As substituições nos casos de ausência dos industriários que não mais trabalhavam na empresa foram efetuadas pelo nome imediatamente anterior da relação nominal em ordem alfabética.

Na determinação do tamanho da amostra, recorreu-se aos procedimentos propostos por Luiz \& Magnanini 15 para populações finitas. Nesse cálculo, foi adotado um nível de significância de $5 \%$ (correspondendo a um intervalo de confiança de $95 \%, z[\alpha] / 2=1,96)$ e erro tolerável de amostragem de $3 \%$, resultando numa amostra necessária de 543 sujeitos para estimativa da prevalência de percepção negativa de saúde em $15 \%$. Essa primeira estimativa de tamanho amostral foi aumentada em $15 \%$, no intuito de explorar associações ajustadas entre percepção de saúde e variáveis independentes, perfazendo uma amostra mínima necessária de 625 trabalhadores. Posteriormente, a amostra foi aumentada em mais $20 \%$ de questionários, a fim de compensar eventuais perdas e recusas. Em virtude de o inquérito contemplar outras variáveis com menores desfechos, o número final de questionários distribuídos para a coleta foi maior (3 mil) que o calculado (750).

Para garantir a padronização da coleta de dados, os gestores do SESI/DR-SC foram treinados pelo coordenador do projeto. Em seguida, cada gestor ficou responsável pela tarefa de solicitar a autorização das empresas para realização do levantamento, providenciar a listagem para realizar o sorteio dos trabalhadores e coletar os dados. Depois, todo o processo de coleta foi acompanhado por plantão de atendimento mantido no Núcleo de Pesquisas em Atividade Física e Saúde da Universidade Federal de Santa Catarina (NuPAF/UFSC).

No levantamento das informações, usou-se questionário previamente validado 13 para essa população. O instrumento não continha campos que permitissem a identificação e, antes de iniciar a aplicação do instrumento, todos foram esclarecidos acerca da garantia de anonimato das informações fornecidas e sobre o caráter voluntário da participação na pesquisa. Em seguida, procedeu-se à aplicação dos questionários em pequenos grupos, exceto para os trabalhadores de baixa escolaridade, que foram entrevistados individualmente. Em ambas as situações, os sujeitos foram continuamente acompanhados pelos aplicadores para esclarecimento de dúvidas.

A percepção de saúde foi mensurada com base na questão "Como você classifica seu estado de saúde atual?", com quatro opções de resposta que foram subseqüentemente dicotomizadas (excelente, bom = percepção positiva , regular, ruim = percepção negativa). A percepção da qualidade do sono foi mensurada por meio da questão: "Com que freqüência você considera que dorme bem?", e a percepção do nível de estresse foi medida pela questão "Como você descreve o nível de estresse em sua vida?”. Ambas tinham quatro opções de resposta. Quanto à demanda física no trabalho, foi formulada a seguinte questão: "Suas atividades no trabalho podem ser descritas como?”. Seguem descrições na Tabela 1.

Doze variáveis independentes foram selecionadas, conforme apresentado na Tabela 1 .

As informações coletadas foram digitalizadas em leitura ótica pelo programa SPHYNX (Sphynx Software Solutions Incorp., Washington DC, Estados Unidos), de forma a evitar erros 
Níveis e critérios de categorização das variáveis independentes.

\begin{tabular}{|c|c|c|}
\hline Nível & Tipo da variável & Critérios de categorização \\
\hline \multirow[t]{5}{*}{ Distal } & Demográfica & Sexo \\
\hline & & Faixa etária (anos) \\
\hline & & $\leq 29$ \\
\hline & & $30-39$ \\
\hline & & $40 \mathrm{ou}+$ \\
\hline \multirow[t]{18}{*}{ Intermediário } & Sócio-econômica & Nível de escolarização \\
\hline & & Fundamental incompleto \\
\hline & & Fundamental completo/Médio incompleto \\
\hline & & Médio completo/Superior incompleto \\
\hline & & Superior completo \\
\hline & & Estado civil \\
\hline & & Solteiro \\
\hline & & Casado/Com parceiro(a) \\
\hline & & Viúvo/Divorciado ou separado(a) \\
\hline & & Renda (em Reais) * \\
\hline & & $\leq 520,00$ \\
\hline & & $521,00-1.300,00$ \\
\hline & & $1.301,00-2.600,00$ \\
\hline & & $>2.600,00$ \\
\hline & & Nível de demanda física no trabalho \\
\hline & & Pouco ativo (maior parte do tempo sentado) \\
\hline & & Moderadamente ativo (caminhar rápido, tarefas manuais) \\
\hline & & Trabalho pesado (atividades físicas intensas) \\
\hline \multirow[t]{14}{*}{ Proximal } & Fatores de risco à saúde & Inatividade física no lazer (referir não realizar atividade física no lazer) \\
\hline & & Fumantes (referir fumar, independente da quantidade) \\
\hline & & Consumo excessivo ocasional de bebida alcoólica (referir ingestão \\
\hline & & $\geq 5$ doses de bebida alcoólicas numa mesma ocasião durante o \\
\hline & & último mês [binge drinking]) \\
\hline & & Índice de massa corporal (kg/m²) \\
\hline & & Baixo peso $(<18,5)$ \\
\hline & & Recomendável $(18,5-24,9)$ \\
\hline & & Sobrepeso $(25,0-29,9)$ \\
\hline & & Obesidade $(+30)$ \\
\hline & & Percepção de negativa do nível de estresse \\
\hline & & Referir nível "quase sempre" ou "sempre estressado" \\
\hline & & Percepção negativa do sono \\
\hline & & Referir dormir bem "às vezes" ou "nunca" \\
\hline
\end{tabular}

* Múltiplos de salário mínimo (R 260,00$)$.

de digitação. Além disso, as informações foram transportadas para o programa Excel for Windows (Microsoft Corp., Estados Unidos) a fim de uniformizar, eletrônica e manualmente, a unidade de medida da estatura (centímetros para metro). A seguir, os dados foram exportados para o programa SPSS, versão 11.0 (SPSS Inc., Chicago, Estados Unidos), com o qual foram realizadas a análise bruta e a análise multivariável pela regressão logística não condicional, com base na abordagem hierárquica em três níveis (Tabela 1) conforme modelo sugerido por Victora et al. 16. Esta proposta de análise ajusta o efeito de cada variável para aquelas num nível igual ou superior. Análises adicionais referentes às diferenças de prevalências foram realizadas por meio do teste crítico de razões (z), utilizando-se, para tanto, o programa estatístico EpiCalc 2000; versão 1.02 (EpiCalc: Epidemiological calculator, http://cran.r-project.org/src/contrib/ 
Descriptions/epicalc.html). Em todas as análises, os resultados foram definidos como estatisticamente significativos para um valor de $\mathrm{p}$ $\leq 0,05$.

O projeto ( $\left.n^{\circ} .305 / 04\right)$ foi aprovado na íntegra pelo Comitê de Ética em Pesquisas com Seres Humanos da UFSC.

\section{Resultados}

A amostra foi composta por 2.574 sujeitos, com maior proporção de homens (62,5\%). Mais da metade dos industriários tinha menos de 30 anos de idade (53\%) e vivia em comunhão estável (62\%). Apesar das semelhanças nos diferentes níveis de escolarização, observou-se pior distribuição de renda entre as mulheres. Como esperado, a execução de trabalho pesado foi maior entre os homens (Tabela 2).

Informações referentes aos fatores de risco revelaram um perfil de risco passivo entre as mulheres (percepção negativa de estresse e inatividade física no lazer) e de risco ativo entre os homens (fumo e álcool). Maiores prevalências de excesso de peso corporal e de percepção negativa do sono também foram verificados para o sexo masculino (Tabela 2). Entretanto, os mesmos apresentaram uma menor prevalência de percepção negativa de saúde em relação às mulheres, para uma prevalência geral de 11,8\%.

Os resultados da Tabela 3 indicam que um quarto das variáveis (estado civil, consumo de bebidas alcoólicas e tabagismo) não demonstrou associação com a percepção de saúde. Dentre as variáveis sócio-demográficas, a prevalência da percepção negativa de saúde foi maior entre industriários que exerciam trabalho pesado, além de estar positivamente associada à faixa etária e inversamente relacionada ao nível de escolarização e a renda familiar. Já em nível proximal, as categorias de variáveis referentes à percepção negativa do sono e do estresse, obesidade, baixo peso e inatividade física no lazer, apresentaram, respectivamente, valores decrescentes de razão de chances.

Diferenças importantes no perfil das associações foram discriminadas em função do sexo e respectivos tipos de análise: bruta (Tabela 4) e multivariável (não ilustrados em tabela). Homens apresentaram perfil semelhante ao observado no geral e, além disso, seus resultados confirmaram a associação entre estado civil e percepção de saúde ( $\mathrm{p}=0,002)$. Já entre as mulheres, foram identificadas associações somente na metade das 12 variáveis investigadas e, mesmo assim, apenas três mantiveram a associação após a análise multivariável: trabalho pesado $(\mathrm{RC}=2,04)$; percepção negativa de estresse $(\mathrm{RC}=3,13)$; percepção negativa de sono $(\mathrm{RC}=3,82)$.

\section{Discussão}

Além das limitações inerentes às investigações de delineamento transversal, este estudo pode apresentar viés de seleção por priorizar as indústrias participantes do inquérito de 1999. Contudo, comparações das características descritivas de ambos os inquéritos (PEVIC I e PEVIC II) 17 não evidenciaram discrepâncias importantes na composição da amostra. Já os resultados do índice de massa corporal (IMC) podem ter sido afetados pelo viés de aferição, pois as informações referentes ao peso e à estatura foram obtidas na forma referida.

Por outro lado, obteve-se uma boa qualidade na entrada dos dados em função do sistema de leitura ótica, além da boa taxa de retorno dos questionários distribuídos (86\%), que resultou em tamanho amostral suficiente para o tratamento estatístico executado. Já o controle do viés de confundimento, baseado na abordagem hierárquica para análise de regressão logística, adicionada de três variáveis referentes ao indicador sócio-econômico (renda familiar, escolaridade e características do trabalho) representam as estratégias metodológicas de maior destaque. $\mathrm{O}$ acréscimo de indicadores sócio-econômicos nesta proposta de investigação é muito importante, visto que evidências sustentam a possibilidade de a iniqüidade social influenciar na percepção de saúde 7,9 e no risco de mortalidade 1 , mesmo em países desenvolvidos.

A prevalência de percepção negativa de saúde $(11,8 \%)$ foi significantemente inferior $(z=3,45$; $\mathrm{p}=0,001)$ à prevalência $(14,8 \%)$ observada para esta mesma população em inquérito realizado há cinco anos 13. O mesmo ocorreu em comparação às prevalências relatadas para amostras brasileiras (Porto Alegre - 18\%; Florianópolis - 21,4\%; Belém - 36\%), norte-americanas (15,5\%), e russas (24\%). Todavia, na Finlândia e em países bálticos (Estônia e Lituânia), as prevalências foram menores (8 e 11\%) em relação aos industriários catarinenses.

Mesmo com a redução de $20 \% 13$ para $13,5 \%$ na prevalência de percepção negativa de saúde entre as industriarias catarinenses, manteve-se a diferença significativa $(\mathrm{p}=0,007)$ entre os sexos neste último inquérito. Porém, por mais que os indicadores relativos à percepção de saúde coincidam com evidências de outras investigações 10,12 , análises paralelas para este grupo de trabalhadores 17 evidenciaram maior simultaneidade de fatores de risco à saúde entre homens. Logo, 
Características demográficas, sócio-econômicas, comportamentais, perceptivas e de saúde em industriários de Santa Catarina, Brasil, 2004.

\begin{tabular}{|c|c|c|c|c|}
\hline Variável & $\begin{array}{c}\text { Homens } \\
\%\end{array}$ & $\begin{array}{c}\text { Mulheres } \\
\%\end{array}$ & $\begin{array}{c}\text { Geral } \\
\%\end{array}$ & $p$ \\
\hline Faixa etária (anos) & $n=1.603$ & $n=960$ & $\mathrm{n}=2.563$ & 0,001 \\
\hline$\leq 29$ & 51,3 & 56,8 & 53,3 & \\
\hline $30-39$ & 31,4 & 30,7 & 31,2 & \\
\hline 40 ou + & 17,3 & 12,5 & 15,5 & \\
\hline Nível de escolarização & $n=1.602$ & $n=960$ & $\mathrm{n}=2.562$ & 0,766 \\
\hline Fundamental incompleto & 15,8 & 16,8 & 16,2 & \\
\hline Fundamental Completo/Médio incompleto & 25,4 & 23,9 & 24,8 & \\
\hline Médio completo/Superior incompleto & 47,7 & 46,8 & 47,3 & \\
\hline Superior completo & 11,1 & 12,6 & 12,7 & \\
\hline Estado civil & $n=1.599$ & $n=960$ & $\mathrm{n}=2.559$ & 0,001 \\
\hline Solteiro & 33,0 & 34,2 & 33,4 & \\
\hline Casado/Com parceiro & 63,9 & 58,8 & 62,0 & \\
\hline Viúvo/Divorciado/Separado & 3,1 & 7,1 & 4,6 & \\
\hline Renda (em Reais) & $n=1.600$ & $\mathrm{n}=955$ & $\mathrm{n}=2.555$ & 0,024 \\
\hline$\leq 520,00$ & 20,0 & 27,1 & 22,7 & \\
\hline $521,00-1.300,00$ & 47,2 & 42,7 & 45,5 & \\
\hline $1.301,00-2.600,00$ & 9,9 & 7,3 & 9,0 & \\
\hline$>2.600,00$ & 22,9 & 22,8 & 22,9 & \\
\hline Características do trabalho & $n=1.593$ & $n=953$ & $\mathrm{n}=2.546$ & 0,001 \\
\hline Pouco ativo & 25,7 & 42,7 & 32,1 & \\
\hline Moderadamente ativo & 29,1 & 48,1 & 55,0 & \\
\hline Trabalho pesado & 15,2 & 9,2 & 13,0 & \\
\hline Atividade física no lazer & $n=1.575$ & $n=926$ & $n=2.501$ & 0,001 \\
\hline Ativo no lazer & 77,2 & 55,6 & 69,2 & \\
\hline Inativo no lazer & 22,8 & 44,4 & 30,8 & \\
\hline Tabagismo & $n=1.502$ & $\mathrm{n}=917$ & $n=2.419$ & 0,001 \\
\hline Não fumantes & 83,7 & 90,4 & 86,2 & \\
\hline Fumantes & 16,3 & 9,6 & 13,8 & \\
\hline Consumo de bebidas alcoólicas & $n=1.502$ & $n=917$ & $n=2.419$ & 0,001 \\
\hline Não excessivo & 49,0 & 75,6 & 59,0 & \\
\hline Excessivo ocasional & 51,0 & 24,4 & 41,0 & \\
\hline Percepção do sono & $n=1.598$ & $n=963$ & $\mathrm{n}=2.561$ & 0,048 \\
\hline Positiva & 84,0 & 86,9 & 85,1 & \\
\hline Negativa & 16,0 & 13,1 & 14,9 & \\
\hline Percepção de estresse & $n=1.595$ & $n=959$ & $\mathrm{n}=2.552$ & 0,001 \\
\hline Positiva & 89,2 & 84,6 & 87,4 & \\
\hline Negativa & 10,8 & 15,4 & 12,6 & \\
\hline Índice de massa corporal $\left(\mathrm{kg} / \mathrm{m}^{2}\right)$ & $n=1.509$ & $n=869$ & $\mathrm{n}=2.378$ & 0,001 \\
\hline Baixo peso $(<18,5)$ & 1,7 & 5,9 & 3,2 & \\
\hline Normal $(18,5-24,9)$ & 55,7 & 67,8 & 60,0 & \\
\hline Sobrepeso $(25,0-29,9)$ & 35,6 & 19,8 & 29,8 & \\
\hline Obesidade $(\geq 30)$ & 7,3 & 6,6 & 7,0 & \\
\hline
\end{tabular}

na interpretação dos dados, deve-se considerar uma aparente divergência no rigor do autoconceito, da concepção e dos cuidados com a saúde entre os sexos, uma vez que, assim como relata Shadbolt 18 , a percepção de saúde entre mulheres reflete um processo complexo que envolve a experiência de vida, presença de doenças crônicas, vitalidade, atitude mental e estilo de vida.

Esses aspectos talvez possam explicar a inexistência de associação para as variáveis faixa 
Prevalência da percepção negativa de saúde e razão de chances (RC) bruta e multivariável para variáveis independentes em relação ao desfecho em industriários de Santa Catarina, Brasil, 2004

\begin{tabular}{|c|c|c|c|c|c|}
\hline \multirow[t]{2}{*}{ Variável } & \multirow{2}{*}{$\begin{array}{l}\text { Percepção negativa } \\
\text { de saúde(\%) }\end{array}$} & \multicolumn{2}{|c|}{ Análise bruta } & \multicolumn{2}{|c|}{ Análise multivariável } \\
\hline & & RC (IC95\%) & $\mathrm{p}$ & RC (IC95\%) & $\mathrm{p}$ \\
\hline Sexo * & & & 0,012 & & 0,007 \\
\hline Masculino & 10,2 & 1,00 & & $1,00 \#$ & \\
\hline Feminino & 13,5 & $1,37(1,07-1,75)$ & & $1,40(1,09-1,80)$ & \\
\hline Faixa etária (anos) * & & & 0,001 & & 0,001 \\
\hline$\leq 29$ & 9,4 & 1,00 & & $1,00 \#$ & \\
\hline $30-39$ & 11,9 & $1,29(0,97-1,71)$ & & $1,31(0,99-1,74)$ & \\
\hline 40 ou + & 17,1 & $1,98(1,44-2,72)$ & & $2,05(1,49-2,83)$ & \\
\hline Nível de escolarização ** & & & 0,001 & & 0,006 \\
\hline Fundamental incompleto & 16,6 & $2,38(1,45-3,92)$ & & $1,80(0,99-3,28)$ & \\
\hline Fundamental completo/Médio incompleto & 14,9 & $2,10(1,30-3,39)$ & & $1,93(1,09-3,39)$ & \\
\hline Médio completo/Superior incompleto & 8,7 & $1,14(0,71-1,83)$ & & $1,17(0,69-1,99)$ & \\
\hline Superior completo & 7,7 & 1,00 & & 1,00 \#\# & \\
\hline 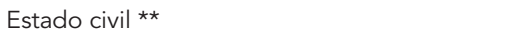 & & & 0,030 & & 0,324 \\
\hline Solteiro & 10,3 & 1,00 & & $1,00 \# \#$ & \\
\hline Casado/Com Parceiro & 11,5 & $1,12(0,86-1,47)$ & & $0,87(0,63-1,20)$ & \\
\hline Viúvo/Divorciado/Separado & 18,6 & $2,00(1,19-3,34)$ & & $1,24(0,70-2,19)$ & \\
\hline Renda (em Reais) $\star \star ~$ & & & 0,001 & & 0,006 \\
\hline$\leq 520,00$ & 16,7 & $1,70(1,05-2,74)$ & & $1,21(0,68-2,14)$ & \\
\hline $521,00-1.300,00$ & 11,0 & $1,04(0,66-1,66)$ & & $0,83(0,49-1,42)$ & \\
\hline $1.301,00-2.600,00$ & 7,4 & $0,67(0,40-1,14)$ & & $0,59(0,34-1,03)$ & \\
\hline$>2.600,00$ & 10,5 & 1,00 & & 1,00 \#\# & \\
\hline Características do trabalho ** & & & 0,001 & & 0,003 \\
\hline Pouco ativo & 10,8 & $1,09(0,82-1,45)$ & & $1,28(0,93-1,74)$ & \\
\hline Moderadamente ativo & 9,9 & 1,00 & & 1,00 \#\# & \\
\hline Trabalho pesado & 19,2 & $2,16(1,56-2,98)$ & & $1,79(1,27-2,53)$ & \\
\hline 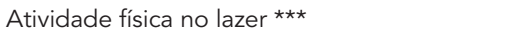 & & & 0,001 & & 0,042 \\
\hline Ativo no lazer & 9,1 & 1,00 & & $1,00 \# \#$ & \\
\hline Inativo no lazer & 15,9 & $1,89(1,46-2,43)$ & & $1,40(1,01-1,93)$ & \\
\hline 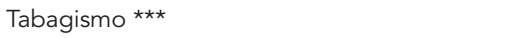 & & & 0,090 & & 0,736 \\
\hline Não fumantes & 10,6 & 1,00 & & 1,00 \#\#\# & \\
\hline Fumantes & 13,8 & $1,34(0,95-1,88)$ & & $0,91(0,59-1,38)$ & \\
\hline 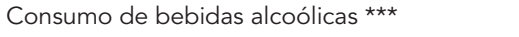 & & & 0,153 & & 0,456 \\
\hline Não excessivo & 12,2 & 1,00 & & 1,00 \#\#\# & \\
\hline Excessivo ocasional & 10,4 & $0,83(0,65-1,07)$ & & $0,88(0,63-1,23)$ & \\
\hline 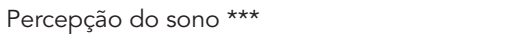 & & & 0,001 & & 0,001 \\
\hline Positiva & 8,8 & 1,00 & & 1,00 \#\#\# & \\
\hline Negativa & 26,2 & $3,67(2,79-4,81)$ & & $3,50(2,50-4,90)$ & \\
\hline 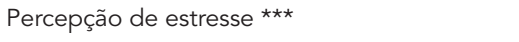 & & & 0,001 & & 0,001 \\
\hline Positiva & 9,2 & 1,00 & & 1,00 \#\#\# & \\
\hline Negativa & 27,4 & $3,72(2,80-4,94)$ & & $2,84(1,98-4,06)$ & \\
\hline 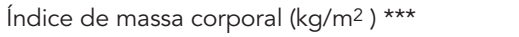 & & & 0,001 & & 0,003 \\
\hline Baixo peso $(<18,5)$ & 16,0 & $1,67(0,88-3,17)$ & & $2,01(1,20-3,38)$ & \\
\hline Normal $(18,5-24,9)$ & 10,2 & 1,00 & & 1,00 \#\#\# & \\
\hline Sobrepeso $(25,0-29,9)$ & 10,0 & $0,97(0,72-1,31)$ & & $0,88(0,61-1,27)$ & \\
\hline Obesidade $(\geq 30)$ & 22,8 & $2,58(1,73-3,86)$ & & $2,36(1,13-4,92)$ & \\
\hline
\end{tabular}

Nível no modelo hierárquico: * nível distal; ** nível intermediário; *** nível proximal;

\# Controlando para variáveis em nível distal;

\#\# Controlando para variáveis em nível distal e intermediário;

\#\# Controlando para variáveis em nível distal, intermediário e proximal;

Número de sujeitos incluídos na análise ao final do 3o nível da abordagem hierárquica = 2.141.

Teste de Hosmer-Lemeshow goodness-of-fit $p=0,993$ 
Prevalência de percepção negativa de saúde e razão de chances (RC) bruta, por sexo, para variáveis independentes em relação ao desfecho em industriários de Santa Catarina, Brasil, 2004

\begin{tabular}{|c|c|c|c|c|c|c|}
\hline \multirow[t]{2}{*}{ Variável } & \multicolumn{3}{|c|}{ Homens } & \multicolumn{3}{|c|}{ Mulheres } \\
\hline & $\begin{array}{l}\text { pção negativa } \\
\text { saúde (\%) }\end{array}$ & RC (IC95\%) & $\mathbf{p}$ & $\begin{array}{c}\text { Percepção negativa } \\
\text { de saúde (\%) }\end{array}$ & RC (IC95\%) & $\mathrm{p}$ \\
\hline Faixa etária (anos) & & & 0,001 & & & 0,359 \\
\hline$\leq 29$ & 7,3 & 1,00 & & 12,7 & 1,00 & \\
\hline $30-39$ & 11,3 & $1,62(1,10-2,37)$ & & 12,9 & $1,02(0,66-1,55)$ & \\
\hline $40 \mathrm{ou}+$ & 17,0 & $2,59(1,72-3,90)$ & & 17,5 & $1,46(0,85-2,49)$ & \\
\hline Nível de escolarização & & & 0,001 & & & 0,006 \\
\hline Fundamental incompleto & 14,6 & $2,17(1,12-4,22)$ & & 19,9 & $2,75(1,29-5,85)$ & \\
\hline Fundamental completo/Médio incompleto & 14,0 & $2,06(1,10-3,88)$ & & 16,6 & $2,20(1,05-4,60)$ & \\
\hline Médio completo/Superior incompleto & 7,5 & $1,02(0,54-1,91)$ & & 10,9 & $1,36(0,66-2,77)$ & \\
\hline Superior completo & 7,3 & 1,00 & & 8,3 & 1,00 & \\
\hline Estado civil & & & 0,001 & & & 0,491 \\
\hline Solteiro & 9,3 & 1,00 & & 11,9 & 1,00 & \\
\hline Casado/Com parceiro & 9,8 & $1,05(0,74-1,51)$ & & 14,5 & $1,26(0,83-1,89)$ & \\
\hline Viúvo/Divorciado/Separado & 28,0 & $3,79(1,91-7,51)$ & & 11,8 & $0,98(0,44-2,22)$ & \\
\hline Renda (em Reais) & & & 0,005 & & & 0,008 \\
\hline$\leq 520,00$ & 14,7 & $1,43(0,79-2,58)$ & & 19,3 & $2,15(0,93-4,98)$ & \\
\hline $521,00-1.300,00$ & 10,2 & $0,94(0,54-1,64)$ & & 12,5 & $1,29(0,56-2,96)$ & \\
\hline $1.301,00-2.600,00$ & 6,3 & $0,56(0,29-1,07)$ & & 9,2 & $0,91(0,37-2,25)$ & \\
\hline$>2.600,00$ & 10,8 & 1,00 & & 10,0 & 1,00 & \\
\hline Característica do trabalho & & & 0,002 & & & 0,001 \\
\hline Pouco ativo & 9,0 & $1,00(0,66-1,50)$ & & 12,5 & $1,07(0,71-1,61)$ & \\
\hline Moderadamente ativo & 9,0 & 1,00 & & 11,8 & 1,00 & \\
\hline Trabalho pesado & 16,5 & $1,99(1,33-2,99)$ & & 27,3 & $2,80(1,62-4,85)$ & \\
\hline Atividade física no lazer & & & 0,001 & & & 0,068 \\
\hline Ativo no lazer & 8,1 & 1,00 & & 11,5 & 1,00 & \\
\hline Inativo no lazer & 16,4 & $2,21(1,56-3,13)$ & & 15,6 & $1,42(0,97-2,08)$ & \\
\hline Tabagismo & & & 0,124 & & & 0,221 \\
\hline Não fumantes & 9,5 & 1,00 & & 12,4 & 1,00 & \\
\hline Fumantes & 12,7 & $1,39(0,91-2,12)$ & & 17,0 & $1,44(0,80-2,62)$ & \\
\hline Consumo de bebidas alcoólicas & & & 0,900 & & & 0,141 \\
\hline Não excessivo & 10,1 & $1,02(0,74-1,41)$ & & 14,4 & $0,71(0,44-1,12)$ & \\
\hline Excessivo ocasional & 10,3 & 1,00 & & 10,6 & 1,00 & \\
\hline Percepção do sono & & & 0,001 & & & 0,001 \\
\hline Positiva & 7,6 & 1,00 & & 10,9 & 1,00 & \\
\hline Negativa & 23,9 & $3,82(2,68-5,43)$ & & 31,0 & $3,67(2,37-5,68)$ & \\
\hline Percepção de estresse & & & 0,001 & & & 0,001 \\
\hline Positiva & 8,5 & 1,00 & & 10,5 & 1,00 & \\
\hline Negativa & 24,9 & $3,55(2,40-5,25)$ & & 30,4 & $3,73(2,46-5,65)$ & \\
\hline Índice de massa corporal (kg/m²) & & & 0,001 & & & 0,023 \\
\hline Baixo peso $(<18,5)$ & 20,8 & $2,59(0,94-7,15)$ & & 13,7 & $1,19(0,52-5,10)$ & \\
\hline Normal $(18,5-24,9)$ & 9,2 & 1,00 & & 11,7 & 1,00 & \\
\hline Sobrepeso $(25,0-29,9)$ & 9,5 & $1,03(0,71-1,50)$ & & 11,6 & $0,99(0,58-1,68)$ & \\
\hline Obesidade $(\geq 30)$ & 20,9 & $2,60(1,55-4,37)$ & & 26,3 & $2,69(1,41-5,10)$ & \\
\hline
\end{tabular}


etária, estado civil, escolaridade e renda familiar com a percepção de saúde entre as mulheres. Já a maior prevalência da percepção negativa de saúde entre homens classificados na categoria viúvo/divorciado/separado, em relação aos solteiros e casados, poderia ser mais bem elucidada por maiores estratificações de categorias da condição matrimonial, pois dados anteriores relativos a este grupo populacional (PEVIC I) 13 demonstraram maior prevalência de percepção negativa de saúde entre viúvos. Não obstante, a condição de viuvez também foi discriminante para o maior risco de percepção negativa de saúde na população russa 19 .

A associação inversa entre percepção de saúde e escolaridade é consistente com informações disponíveis na literatura nacional 6,13 e internacional 1,19. Mais importante, deve-se destacar a maior prevalência de percepção negativa de saúde nas pessoas com menos de oito anos de estudo (geral e homens), fato que também foi estatisticamente significativo para todas as capitais brasileiras investigadas no último inquérito nacional 6

Adicionalmente, as conseqüências da iniqüidade social também foram reveladas nas maiores prevalências de negativa de saúde entre industriários de menor renda e de tarefas laborais de maior demanda física. Essa situação parece ser suportada pelas hipóteses levantadas por Warren et al. 7, que constataram que as características físicas e psicossociais do trabalho exercem papel mediador na relação entre o nível sócio-econômico e a saúde.

Ainda que considerando diferenças de instrumentos e cortes para mensurar atividade física de lazer, a relação inversa desta variável com a percepção de saúde converge com achado nacional 8 e canadense 9 . Para este último grupo, Segovia et al. 9 demonstraram que maior engajamento em atividades físicas de lazer era acompanhado de melhores níveis de percepção de saúde.

Contrariamente aos achados de outros trabalhos 5,9, o hábito de fumar não indicou associação com a percepção de saúde. Em contrapartida, confirmou achados da primeira investigação realizada com esta população ${ }^{8}$ de trabalhadores no PEVIC I - 1999, e com resultados observados para população russa 19. Possivelmente, análise incluindo maior número de categorias para identificar a quantidade de cigarros possa melhor discriminar tal relação.

Assim como nos resultados de outras investigações 5,8, não foi constatada associação entre consumo de bebida alcoólica e percepção de saúde. Sabe-se, no entanto, que além do viés de memória, os critérios de mensuração desta vari- ável podem influenciar nos resultados. O critério adotado no presente estudo (binge drinking) também foi utilizado por Segovia et al. ${ }^{9}$, mediante a combinação do binge drinking com o consumo semanal de bebidas alcoólicas. Por meio dessa estratégia, os autores verificam melhores percepções de saúde em pessoas com consumo moderado de álcool (4 a 7 doses/semana + binge drinking) em relação àquelas pessoas com consumo baixo ou abusivo de bebidas alcoólicas (mais binge drinking).

Similarmente aos achados observados em canadenses 9 , os homens obesos apresentaram maior prevalência de percepção negativa de saúde quando comparados às pessoas na faixa de peso recomendável. Essa informação talvez possa refletir percepções sobre a auto-imagem e da auto-estima dos industriários, que representam um importante papel mediador na adoção de estilo de vida saudável.

Informações disponíveis na literatura acerca da associação entre sono e percepção de saúde referem-se tanto à qualidade 5 quanto à quantidade 9 do sono, revelando melhor percepção de saúde nos sujeitos que reportam sete a oito horas diárias de sono. Esses indicativos também ficaram claros no presente trabalho, em que mesmo após ajustes, a percepção negativa de sono apresentou o maior valor de razão de chances $(\mathrm{RC}=$ 3,48; IC95\%: 2,51-4,82) para percepção negativa de saúde em comparação as demais variáveis investigadas.

A importante associação entre percepção dos níveis de estresse e saúde, relatados por Barros \& Nahas ${ }^{8}$ para este grupo populacional, confirmou-se no presente estudo. Porém, apesar desta constatação, parece existir uma carência de estudos explorando esta relação entre industriários. Somando-se isso aos valores aqui revelados, fazem-se necessárias investigações focadas na inter-relação da percepção de saúde com determinantes físicos e psicossociais do estresse em trabalhadores.

Concluindo, a prevalência da percepção negativa de saúde é baixa e com poucas distinções nas variáveis associadas em comparação a outros estudos. Com base na análise geral ajustada para fatores de confundimento, verificou-se que o desfecho foi positivamente associado com a faixa etária, atividade física de lazer, percepção da qualidade do sono, percepção do estresse e com o sexo feminino. Em contrapartida, a renda familiar bruta e o nível de escolaridade associaram-se inversamente à percepção negativa de saúde. Industriários que exerciam trabalhos com maior demanda física, e aqueles que foram classificados nos extremos das categorias do IMC $\left(<18,5 \mathrm{~kg} / \mathrm{m}^{2} \mathrm{ou} \geq 30 \mathrm{~kg} / \mathrm{m}^{2}\right)$, apresentaram 
maiores prevalências de percepção negativa de saúde.

Diferenças significativas de acordo com o estado civil, consumo de bebidas alcoólicas (binge drinking) e tabagismo não foram verificadas. Além disso, foram reveladas poucas as- sociações para as variáveis investigadas entre o sexo feminino.

Em razão do perfil dissociado verificado entre os sexos, sugere-se a realização de investigações qualitativas acerca da concepção de saúde para triangular com informações quantitativas.

\section{Resumo}

O objetivo foi identificar a prevalência e os fatores associados à percepção negativa de saúde em trabalhadores da indústria no Estado de Santa Catarina, Brasil. Para tanto, foi realizado estudo transversal em amostra representativa de 2.574 sujeitos (62,5\% - homens). A percepção negativa de saúde foi o desfecho investigado em relação a variáveis demográficas, sócio-econômicas, perceptivas (estresse e sono) e de saúde. Análise de regressão logística multivariável não condicional baseada em modelo hierárquico foi utilizada para explorar associações. A percepção negativa de saúde foi baixa $(11,8 \%)$ e positivamente associada com a faixa etária, atividade física de lazer, percepção da qualidade do sono, percepção do estresse e sexo. Em contrapartida, a renda familiar bruta e o nível de escolaridade associaram-se inversamente ao desfecho. Industriários que exerciam trabalhos de maior demanda física e aqueles classificados nos extremos das categorias do índice de massa corporal ( $<18,5 \mathrm{~kg} / \mathrm{m}^{2}$ ou $\left.\geq 30 \mathrm{~kg} / \mathrm{m}^{2}\right)$ apresentaram maiores prevalências de percepção negativa de saúde. Diferenças significativas de acordo com o estado civil, consumo de bebidas alcoólicas (binge drinking) $e$ tabagismo não foram verificadas. Poucas associações foram reveladas para o sexo feminino.

Saúde do Trabalhador; Fatores de Risco; Percepção

\section{Colaboradores}

S. A Fonseca foi o responsável pela análise de dados, delineamento e redação do artigo. V. L. G. Blank colaborou na determinação dos objetivos e na revisão do manuscrito. M.V. G. Barros colaborou na revisão do manuscrito e na elaboração do instrumento de medida. M. V. Nahas colaborou na determinação dos objetivos, delineamento do estudo, revisão do manuscrito e elaboração do instrumento de medida.

\section{Agradecimentos}

Ao Serviço Social da Indústria, Departamento Regional de Santa Catarina (SESI/DR-SC) pelo apoio logístico e institucional para a realização do estudo Perfil do Estilo de Vida e Hábitos de Lazer dos Industriários Catarinenses II. Ao Núcleo de Pesquisa em Atividade Física e Saúde da Universidade Federal de Santa Catarina (NuPAF/ UFSC), pelo suporte logístico na fase de coleta. 


\section{Referências}

1. Sundquist J, Johansson SE. Self reported poor health and low educational level predictors for mortality: a population based follow up study of 39,156 people in Sweden. J Epidemiol Community Health 1997; 51:35-40.

2. Idler EL, Angel RJ. Self-rated health and mortality in the NHANES-I. Epidemiologic Follow-up-study. Am J Public Health 1990; 80:446-52.

3. Reime B, Novak P, Born J, Hagel E, Vanek W. Eating habits, health status, and concern about health: a study among 1641 employees in the german metal industry. Prev Med 2000; 30:295-301.

4. Monden CW, van Lenthe F, de Graaf ND, Kraavkamp G. Partner's and own education: does who you live with matter for self-assessed health, smoking and excessive alcohol consumption? Soc Sci Med 2003; 57:1901-12.

5. Contovannis P, Jones AM. Socio-economic status, health and lifestyle. J Health Econ 2004; 23:965-95.

6. Coordenação de Prevenção e Vigilância, Instituto Nacional de Câncer. Inquérito domiciliar sobre comportamentos de risco e morbidade referida de doenças e agravos não-transmissíveis: Brasil, 15 capitais e Distrito Federal, 2002-2003. Rio de Janeiro: Instituto Nacional de Câncer; 2004.

7. Warren JR, Hoonakker P, Caravon P, Brand J. Job characteristics as mediators in SES-health relationships. Soc Sci Med 2004; 59:1367-78.

8. Barros MVG, Nahas MV. Comportamento de risco, auto-avaliação do nível de saúde e percepção de estresse entre trabalhadores da indústria. Rev Saúde Pública 2001; 35:554-63.

9. Segovia J, Bartlett RF, Edwards AC. The association between self-assessed health status and individual health practices. Can J Public Health 1989; 80:32-7.

10. Kasmel A, Helasoja V, Lipand A, Prattala R, Klumbiene J, Pudule I. Association between health behaviour and self-reported health in Estonia, Finland, Latvia and Lithuania. Eur J Public Health 2004; 14:32-6.
11. Kaplan GA, Camacho T. Perceived health and mortality: a nine-year follow-up of the human population laboratory cohort. Am J Epidemiol 1983; 117:292-304.

12. Zahran HS, Kobau R, Moriarty DG, Zack MM, Holt J, Donehoo R: Centers for Disease Control and Prevention (CDC). Health-related quality of life surveillance - United States, 1993-2002. MMWR Surveill Summ 2005; 54:1-35.

13. Barros MVG. Atividades físicas no lazer e outros comportamentos relacionados à saúde dos trabalhadores da indústria no Estado de Santa Catarina, Brasil [Dissertação de Mestrado]. Florianópolis: Centro de Desportos, Universidade Federal de Santa Catarina; 1999.

14. Torres TZG. Amostragem. In: Medronho RA, organizador. Epidemiologia. São Paulo. Editora Atheneu; 2006. p. 283-94.

15. Luiz RR, Magnanini MF. A lógica da determinação do tamanho da amostra em investigações epidemiológicas. Cad Saúde Coletiva (Rio J) 2000; 8:9-28.

16. Victora CG, Huttly SR, Fuchs SC, Olinto MTA. The role of conceptual frameworks in epidemiological analysis: a hierarchical approach. Int J Epidemiol 1997; 26:224-7.

17. Fonseca SA. Inatividade física no lazer e outros fatores de risco à saúde em industriários catarinenses, 1999-2004 [Dissertação de Mestrado]. Florianópolis: Centro de Desportos, Universidade Federal de Santa Catarina; 2005.

18. Shadbolt B. Some correlates of self-rated for Australian women. Am J Public Health 1997; 87:951-6.

19. Bobak M, Pikhart H, Hertzman C, Rose R, Marmot M. Socioeconomic factors, perceived control and self-reported health in Russia. A cross-sectional survey. Soc Sci Med 1998; 47:269-79.

Recebido em 26/Jul/2006

Versão final reapresentada em 04/Set/2007

Aprovado em 27/Set/2007 